\title{
Enhancement of MTC Performance in LTE Networks by Maximizing Random Access Procedure Throughput
}

\author{
Ibraheem M. Fayed and Eman S. El-Din \\ Network Planning Department, National Telecommunication Institute, Cairo 11768, Egypt
}

\begin{abstract}
A fundamental requirement for any cellular system is the possibility for the device to request a connection setup, commonly referred to as random access procedure. In LTE (long term evolution) networks, the distribution of a limited number of radio resources among H2H (Human-to-Human) users and increasing number of MTC (Machine-Type-Communication) devices in M2M (Machine-to-Machine) communications is one of the main problems. An analytical model is conducted to compute the throughput for message 1 and message 2. This is done using a Markov chain model for the four messages signaling flow with buffering for message 4 . This model is used in LTE 3GPP (Third-Generation Partnership Project) random access. The network performance will be enhanced by determining a dedicated arrival rate corresponding to maximum throughput of message 2 that will assist the network planner to optimize the network performance. In this paper, a deduced arrival rate less than 3.333 requests/ms will maximize network throughput.
\end{abstract}

Key words: MTC (machine type communication), M2M, LTE network, random access procedure.

\section{Introduction}

In the past, mobile communications were limited to the rich people and some people who had special reasons only. Now it is available to the majority of the world's population [1]. Modern wireless communication schemes like 3GPP (Third-Generation Partnership Project) LTE (long term evolution) network, do not allow serving effectively M2M (Machine-to-Machine) connections between a huge numbers of interacting MTC devices. A good background of this issue "when a huge number of machines request access" was explained in Ref. [2]. The finance approach is an important factor to increasing LTE network performance which is a problem facing the network planners. Random Access Procedure $(\mathrm{RACH})$ is a possible solution for this problem [3].

Increasing network capacity is the major objectives of LTE network. By this increasing user will benefit from high data rates usage with low latency, trust

Corresponding author: Ibraheem Mhmoud Fayed, assistant professor, research fields: telecommunications, mobile communications systems, LTE networks. improved QoS (Quality of Service), and bring down the cost. For accessing a channel and trusting the QoS, two attributes of RACH procedures in LTE must be considered: contention based $\mathrm{RACH}$ and contention free RACH.

In contention based RACH, all MSs (mobile stations) request to share the contention for the resources. In contention free RACH, the e-NodeB assigns a reserved preamble for the MS, this will obviate the contention [4].

A very huge number of machines, such as UE (user equipment) may dwell in coverage area of cell, which request periodically to access the network. They must have the minimum power required to be used efficiently as possible. The 3GPP has been carried in different studies $[5,6]$ that try to solve the subjects and problems related to MTC in the existing systems and the future LTE releases too. A detailed study and analysis of PDCCH (physical downlink control channel) performance for M2M traffic in LTE was introduced in Ref. [7].

The main aim of this paper is to obtain the upper band of the request arrival rate in order to sustain the network performance metric such as the throughput for 
MTC in LTE network. An explicit analysis for evaluation of throughput for message 1 (Msg. 1) and message 2 (Msg. 2) is presented. This is done using a Markov chain model for the four messages signaling flow with buffering for message 4 (Msg. 4) in LTE 3GPP random access.

This paper is organized as follows: random access procedure using four messages in LTE network is explained in Section 2. Markov chain model for transient states for the four messages with queuing buffer for Msg. 4 and model assumptions and analysis is introduced in Section 3. In Section 4, results and verification are shown. Section 5 is devoted to conclusion.

\section{Random Access Procedure}

A brief description of the contention based RACH procedure by the MTC channels is explained as follow.

Step A. The MTC device (UE) requests to initiate the $\mathrm{RACH}$ procedure by selecting an $\mathrm{RACH}$ preamble available randomly then sending this preamble in Msg. 1 using the PRACH (physical random access channel) in the uplink. When more than one machine (MS) take the same preamble from a certain sub-frame a collision occurs. However, after preamble was sent, the UE waits for a RAR (random access response). Msg. 2 from the e-NodeB within the time interval called a response window [3], i.e., if more than one MS use the same preamble for Msg. 1 and a collision occurs; the e-NodeB will detect this event and will not send reply to UE. The transmission of an RACH preamble is related to dedicated sub-frames. The random access probably occurs every $b$ sub-frame. So, $b$ refers to periodicity. $K$ refers to the available preambles total number.

Step B. e-NodeB will reply with Msg. 2, known as the RAR and it has UL grant for Step C. Msg. 2 is sent over the physical PDSCH. So, schedule the user is needed [7], i.e., sends a downlink assignment control message over the PDCCH. At most an RAR message in each sub-frame will exist but each sub-frame may have more than one UL grant (each referring to a different preamble). Let $c$ be defined as the maximum number of UL grants per RAR per sub-frame. Note that in this model, a UL grant is given for every uncollided preamble.

Step C. The UE sends Msg. 3 over the PUSCH. The collisions in Step A will appear in Step C: The two or more UEs that take the same preamble in Step A will all try to access the same UL grant in Step C to send their Msg. 3's. As a result, interference will occur and none of the UEs involved will send the subsequent Message 4. In the case of successful preamble transmission after receiving Msg. 2 from the e-NodeB and RAR processing time, the UE sends an RRC connection request Msg. 3 to the e-NodeB over PUSCH (Physical Uplink Shared Channel).

Step D. RACH procedure is completed after the UE receiving a contention resolution message Msg. 4 from the e-NodeB. HARQ (Hybrid automatic repeat request) procedure guarantees a successful transmission of Msg. 3/Msg. 4. HARQ procedure provides a limit in Msg. 3/Msg. 4 sequential transmission attempts. If the limit is reached UE should start a new RACH procedure by sending a preamble.

Let $N$ be defined as the PDCCH resource size (in control channel elements CCEs), $N^{\mathrm{Msg} .2}$ and $N^{\mathrm{Msg} .4}$ are the number of CCEs used to send a Msg. 2 and a Msg. 4, respectively. So, a maximum of $M=N / N^{\mathrm{Msg} .4} \mathrm{Msg}$. 4's can be sent in one sub-frame if Msg. 2 is not present in that sub-frame [7]. When a Msg. 2 is sent in a sub-frame, then at most $m=\left(N-N^{\mathrm{Msg} .2}\right) / N^{\mathrm{Msg} .4} \mathrm{Msg}$. 4's can be sent in that sub-frame.

Although the parameters $N, N^{\mathrm{Msg} .2}$, and $N^{\mathrm{Msg} .4}$ are closely connected from the point of view of the system, this model will be simplified by using the derived parameters $M$ and $m$. This scheme is shown in Fig. 1 .

\section{Model Parameters and Assumptions}

In LTE there are $K$ parallel Aloha channels used, and each time a UE makes a request of retransmission, the preamble is selected in random. By this random selection, 


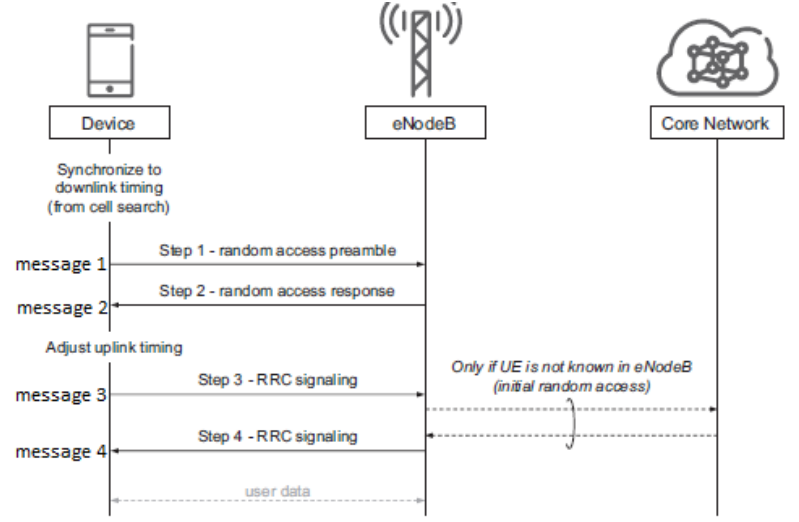

Fig. 1 Four messages control signaling flow sequence in LTE random access [1].

the fresh random access requests and the retransmission attempts will be mixed. In addition, the model input parameter is $a$, which is the aggregate rate of total requests (arrival of new requests and retransmission requests) not equal to $\lambda$ that implies the fresh requests rate per sub-frame.

Let $\theta$ be defined as the throughput of successful requests. When the system is stable, it can be expressed as a function of $a$. If the average input rate is equal to the average output rate the system will be stable, which implies that the fresh requests arrival rate $\lambda$ of is equal to the throughput $\theta$ of successful requests when the system is stable. By this way, the aggregate request rate $a$ and the arrival rate $\lambda$ of fresh requests may be related.

Let $A_{n k}$ be referred to the total number of RACH requests with preamble number $k$ (including the new requests and the requests of retransmissions) in time slot $n$. Since the aggregate stream of requests is assumed to obey a Poisson process and the preambles are independently chosen from the uniform distribution, $A_{n k}$ are IID (Identically Independent Distributed) random variables obeying a Poisson distribution with mean $a b / K$ and point probabilities, i.e.,

$$
P_{\mathrm{i}}(a)=P_{\mathrm{r}}\left\{A_{\mathrm{nk}}=i\right\}=\frac{\left(\frac{a b}{k}\right)^{i}}{i !} e^{-\mathrm{ab} / \mathrm{K}}
$$

The probability of failure can be calculated as follow:

$\mathrm{P}_{\mathrm{r}}\{$ failure $\}=\mathrm{P}_{\mathrm{r}}$ \{collision occurs in Step $\left.\mathrm{A}\right\}+\mathrm{P}_{\mathrm{r}}$ no collision occurs in Step A, loss occurs in Step B $\}+$
$\mathrm{P}_{\mathrm{r}}$ \{no failure occurs in Steps A and B, delay occurs in Step D .

A call flow for successful and unsuccessful session setup establishment based on RACH procedure is shown in Fig. 2.

Utilizing the definition of $q_{\mathrm{ij}}^{(2)}(a)$, we find that:

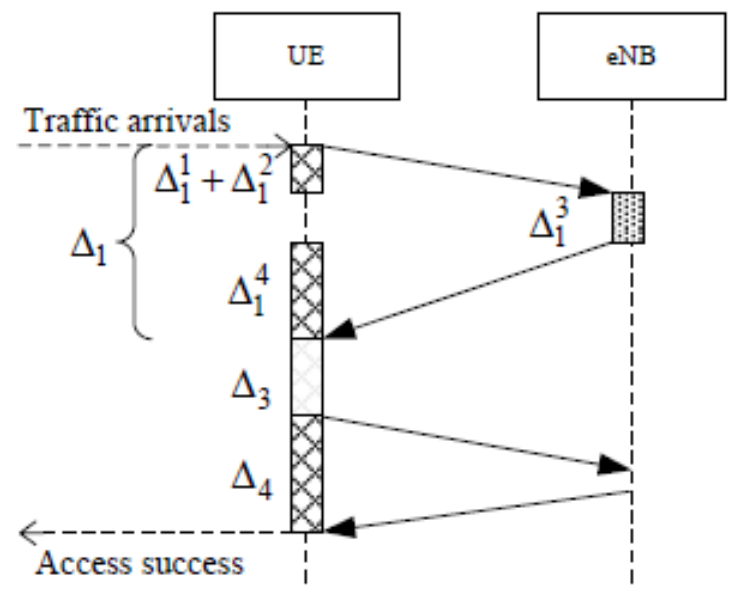

Fig. 2a Request access success without collision.

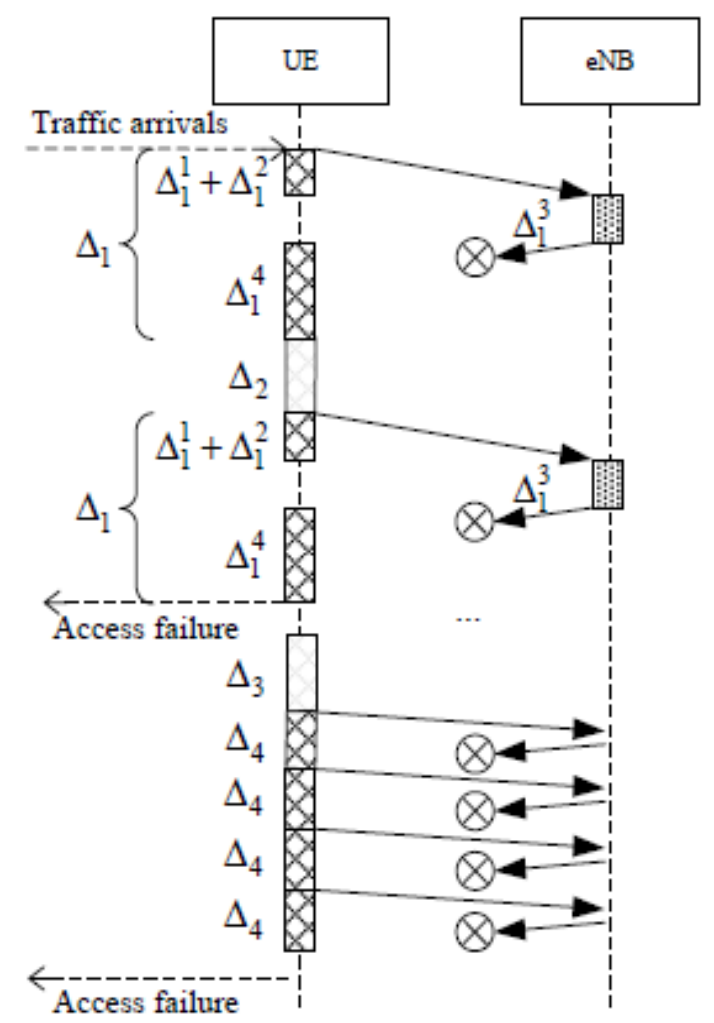

Fig. 2b Request access failure.

Fig. 2 Four messages control signaling flow sequence to transmit data with retransmissions in LTE random access [3]. 


$$
\begin{aligned}
& \tilde{q}_{j}^{(2)}(a) \\
& =\left\{\begin{array}{l}
\left(\begin{array}{c}
K \\
j
\end{array}\right) p_{0}(a)^{K-j}\left(1-p_{0}(a)\right)^{j}, 0 \leq j<b c \\
\sum_{l=b c}^{K}\left(\begin{array}{l}
k \\
l
\end{array}\right) p_{0}(a)^{K-l}\left(1-p_{0}(a)\right)^{l}, j=b c
\end{array}\right.
\end{aligned}
$$

So, $\tilde{Y}_{n}^{(2)}=\min \{B(a), b c\}$.

$B(a)$ refers to a binomially distributed random variable which has parameters $K$ and $l-p_{0}(a)$.

For throughput analysis, the throughput $\theta$ of successful requests and the arrival rate $\lambda$ of fresh requests must be equaled.

This proposal finds conditions for stability in terms of the total traffic $a$ and then to determine the throughput of successful requests $\theta(a)$ as a function of $a$, as well as the maximum throughput $\theta^{*}=\max _{\mathrm{a}} \theta(a)$. To simplify the notation, we assume here that $K>b c$. The generalization to the case $K \leq b c$ is straightforward.

By using the Slotted Aloha model in Ref. [8] for the RACH used in Step 1 the throughput (per sub-frame) of successful Msg. 1's as a function of $a$, which is the arrival rate of all random access requests per sub-frame, can be calculated as follows:

$$
\theta^{(1)}(a)=\frac{E\left[Y_{n}^{(1)}\right]}{b}=a e^{-\frac{a b}{K}}
$$

To calculate the throughput in Step 2, since $K>b c$, the throughput is then reduced by the limited number of UL grants in Msg. 2. The throughput (per sub-frame) of successful UL grants as a function of $a$ is distinct:

$$
\theta^{(2)}(a)=\frac{E\left[Y_{n}^{(2)}\right]}{b}=\frac{1}{b} \sum_{i=1}^{b c} i q_{i}^{(2)}(a)
$$

Eqs. (3) and (4) can be solved using MATLAB software to show the throughput of Msg. 1 and throughput of Msg. 2.

\section{Results Analysis and Verification}

Typical values for the model parameters are taken from Refs. [7, 9] and summarized in Table 1.
Using the above values in Eqs. (1)-(4) and solving using MATLAB program which is explained by the flowing flowchart in Fig. 3, we get the results shown in Figs. 4-6.

As shown in Fig. 4 the arrival rate for requests increases the throughput of Msg. 1 and increases till a certain value $\left(\theta^{(1)}(a)=3.973\right)$ at arrival rate $a=10.67$ requests $/ \mathrm{m}$. Then the throughput approximately sets at this value because the UE will Table 1 Typical values for the model parameters.

\begin{tabular}{lll}
\hline Notion & Definition & Typical values \\
\hline $\mathrm{K}$ & Number of preambles & 54 \\
$\mathrm{~B}$ & RACH Periodicity & 5 \\
$\mathrm{C}$ & $\begin{array}{l}\text { Maximum number of UL } \\
\text { grants per sub-frame }\end{array}$ & 3 \\
\hline
\end{tabular}

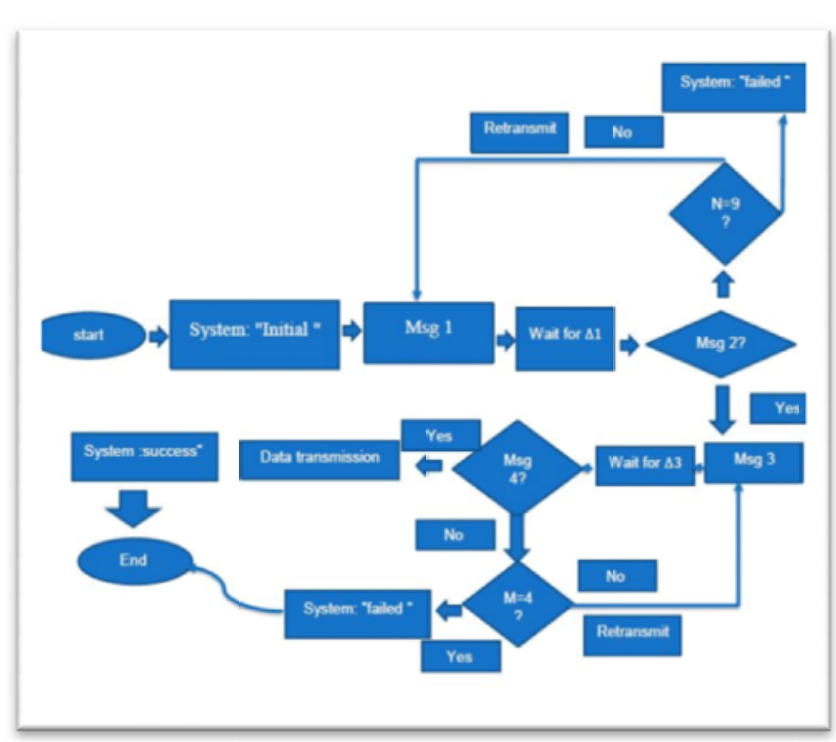

Fig. 3 Flow chart explains the steps used in MATLAP program.

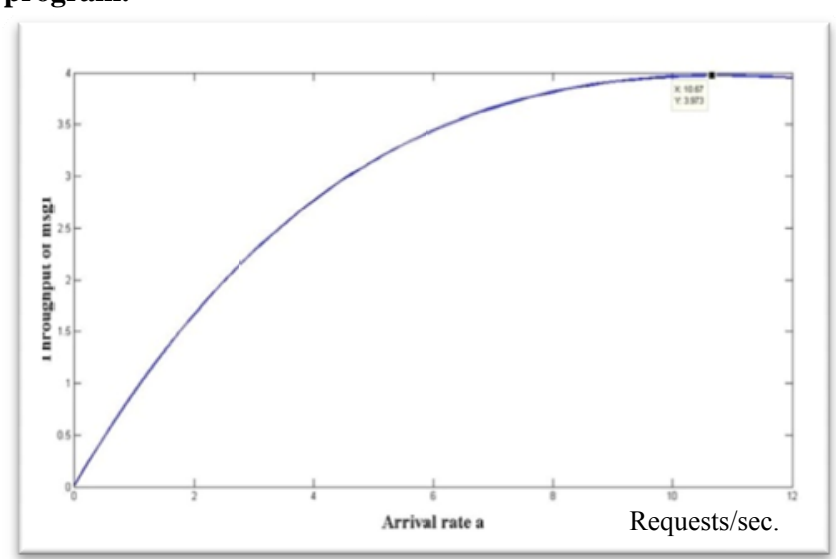

Fig. 4 The throughput of Msg. 1 with the increasing of the aggregate arrival rate $a$ in request/second. 
know after a certain window time that the collision occur and retransmits Msg. 1 again directly with new preamble in the uplink.

As shown in Fig. 5, the arrival rate for requests increases the throughput of Msg. 1 and increases till a certain value $\left(\theta^{(2)}(\mathrm{a})=2.377\right)$ at arrival rate $a=$ 3.333 requests $/ \mathrm{ms}$ and then throughput decreases because the Msg. 2 contains information for more than one user up to 8 users and in case of collision lose downlink for Msg. 2 the all users in this control channel will retransmit (requests) Msg. 1 again directly and may have the same preamble and collision in Msg. 1 dose again.

The failure in Msg. 2 affects directly Msg. 1 so as to get the maximum throughput of Msg. 1 and Msg. 2, the intersection between throughput curve of Msg. 2 and Msg. 1 (which is the maximum throughput for Msg. 2) will be considered as shown in Fig. 6 .

As shown in Fig. 6, the maximum throughput is equal to 2.332 at arrival rate $a$ equals $a=3.333$

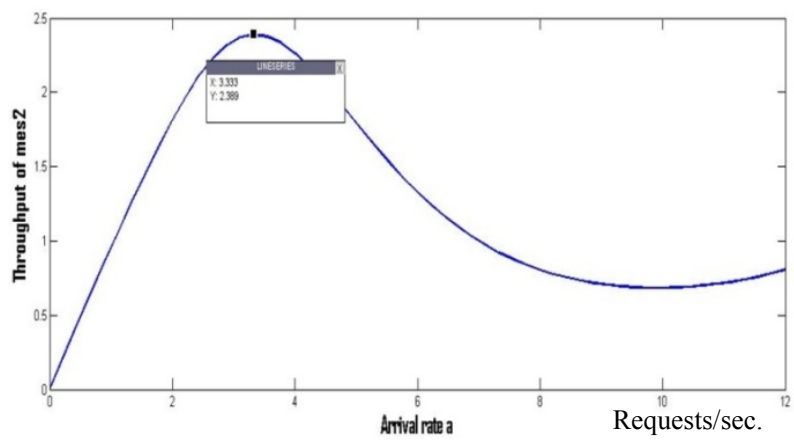

Fig. 5 The throughput of Msg. 2 with the increasing of the aggregate arrival rate $a$ in request/second.

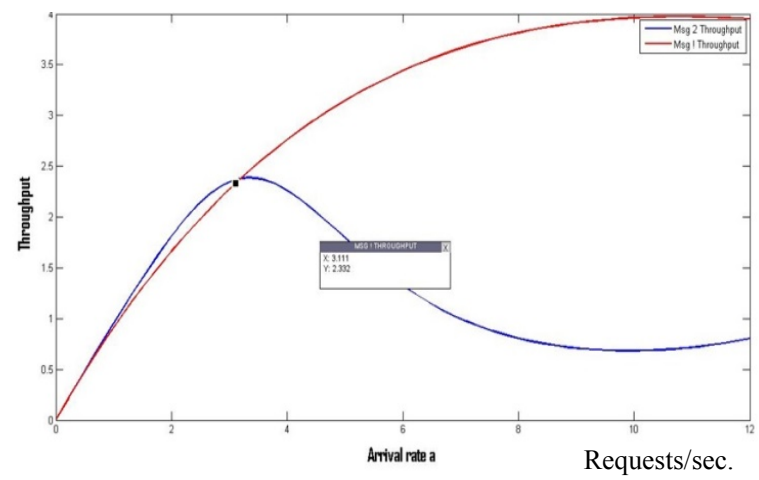

Fig. 6 The intersection between throughput of Msg. 1 and throughput of Msg. 2 with the increasing of the aggregate arrival rate $a$ in request/second. requests/ms and this result is in agreement with Ref. [7].

\section{Conclusions}

The main aim of this paper is to obtain the upper band of the arrival request rate in order to sustain the network performance metric such as the throughput for MTC in LTE network. Explicit analysis for evaluation of throughput for Msg. 1 and Msg. 2 using a Markov chain model for the four messages signaling flow with buffering for Msg. 4 in LTE 3GPP random access was presented.

In LTE planning, it is important to take into consideration the requests arrival rate (as forecasting plan) and the planner must select the network parameters (such as the base station locations, antennas height, etc.) to achieve the maximum throughput. So in this paper, it was found that, the planner has to design the system with arrival rate less than 3.333 requests/ $\mathrm{ms}$ to maximize network throughput. If the arrival rate increases above this value, the collisions in Msg. 1 will increase, causing a degradation of the network throughput and also a decrease of the network performance.

The proposed analytical model will be assigned as a future work to calculate the state probability by using the Markov chain model explained in the proposed model taken into consideration the channel model, distribution and type of users.

\section{References}

[1] Dahlman, E., Parkvall, S., and Skold, J. 2016. 4G, LTE-Advanced Pro and The Road to 5G, 3rd edition, London, United Kingdom: Academic Press, Elsevier.

[2] Lien, S. Y., Chen, K. C., and Lin, Y. 2011. "Toward Ubiquitous Massive Accesses in 3GPP Machine-to-Machine Communications." IEEE Commun. Mag. 49 (4): 66-74.

[3] Borodakiy, V. Y., Samouylov, K. E., Gaidamaka, Y. V., Abaev, P. O., Buturlin, I. A., and Etezov, S. A. 2014. "Modelling a Random Access Channel with Collisions for M2M Traffic in LTE Networks." The Reported Study was Partially Supported by the RFBR, Research Projects No. 13-07-00953, 14-07-00090. 
[4] Khan, N., Khalid, J., and Qureshi, H. K. 2013. "Performance Analysis of Contention-Based Random Access Procedure in Clustered LTE Networks." In Proceedings of the Seventh International Conference on Next Generation Mobile Apps, Services and Technologies, pp. 203-9.

[5] 3GPP R2 112863. 2011. "Backoff Enhancements for RAN Overload Control.” ZTE, Barcelona, Spain, May 2011.

[6] 3GPP TR 23.888 V1.6.0, Third-Generation Partnership Project; Technical Specification Group Services and
System Aspects; System Improvements for Machine-Type Communications (MTC), Sophia-Antipolis, France, Nov. 2011.

[7] Osti, P., Lassila, P., Aalto, S., Larmo, A., and Tirronen, T. 2014. "Analysis of PDCCH Performance for M2M Traffic in LTE." IEEE Transactions on Vehicular Technology 63 (9): 4357-71.

[8] Bertsekas, D., and Gallager, R. 1992. Data Networks, 2nd ed. Upper Saddle River, NJ: Prentice-Hall.

[9] 3GPP TR 37.868. 2011. "Study on RAN Improvements for Machine-Type Communications.” Re-lease 11. 